\title{
Aristocrate malgré lui, ou les mésaventures d'un père d'émigré (1792-1805)
}

\section{Frédérique Pitou}

\section{(2) OpenEdition \\ 1 Journals}

\section{Édition électronique}

URL : https://journals.openedition.org/ahrf/326

DOI : 10.4000 /ahrf.326

ISSN : 1952-403X

Éditeur :

Armand Colin, Société des études robespierristes

\section{Édition imprimée}

Date de publication : 1 mars 2001

Pagination : 35-55

ISSN : 0003-4436

\section{Référence électronique}

Frédérique Pitou, «Aristocrate malgré lui, ou les mésaventures d'un père d'émigré (1792-1805) »,

Annales historiques de la Révolution française [En ligne], 323 | janvier-mars 2001, mis en ligne le 16 mars 2006, consulté le 23 avril 2022. URL : http://journals.openedition.org/ahrf/326 ; DOI : https://doi.org/ 10.4000/ahrf.326

Ce document a été généré automatiquement le 23 avril 2022.

Tous droits réservés 


\title{
Aristocrate malgré lui, ou les mésaventures d'un père d'émigré (1792-1805)
}

\author{
Frédérique Pitou
}

\begin{abstract}
«Á une peine en succède bientôt une autre, on n'a pas sitôt évité une tempête qu'on est accueilli d'un nouvel orage. Lamer en courroux ne s'apaise dans un endroit que pour soulever les flots avec fureur dans un autre $»{ }^{1}$
\end{abstract}

1 Ces considérations sur l'instabilité des choses humaines qui mènent l'individu à s'en remettre aux desseins de la Providence, sont extrêmement fréquentes dans la correspondance des Hardy de Lévaré, famille de notables lavallois vouée depuis des générations à la magistrature et à l'Église. Pendant la Révolution, l'un de ses représentants, René Hardy, a pu en faire l'expérience en 1789, cet avocat de cinquante ans, riche et considéré, fait partie des " premiers habitants » de la ville sa vie, celle de son épouse et celle de ses enfants basculent, non tant avec la Révolution qu'avec l'émigration de son fils aîné, en 1792. S'en suivent une succession de malheurs, emprisonnement, mise sous scellés de ses meubles, séquestre de ses biens fonciers, que l'on peut reconstituer grâce à une série de plus d'une centaine de lettres qui lui sont adressées, conservées avec les brouillons des mémoires et des pétitions qu'il a rédigés ${ }^{2}$. Le réseau de correspondants que se constitue René Hardy entre 1795 et 1800 est composé essentiellement d'avocats de "l'ancien barreau» qui semblent retrouver facilement, dans ces combats de procédure, une culture juridique et des réflexes qu'ils mettent visiblement au service d'autres clients ou amis rencontrant les mêmes soucis. Toute l'énergie de Hardy et de ses correspondants parisiens est consacrée à y voir clair dans la législation mouvante concernant les parents d'émigrés et à choisir la stratégie la plus efficace. Ces documents ne font pas une grande part aux opinions ni aux états d'âme; les allusions aux drames subis par les uns et les autres et aux espoirs entrevus sont fugitives; les prises de position politiques sont absentes, mais le ton global de la correspondance est celui de la déploration. Face à ces hommes de loi, dont les réseaux 
tentent de se reconstituer, l'administration apparait un peu comme un pouvoir autonome, avec sa hiérarchie et ses rites qu'il importe de connaitre et de respecter pour obtenir satisfaction. Tous adressés à Hardy, ou émanant de lui, ces lettres et ces mémoires permettent d'évoquer sa traversée de la Révolution.

Un notable d'Ancien Régime

2 L'exercice généalogique semble la règle parmi les familles de notables lavallois pour la famille Hardy, on dispose de celle qui a été rédigée par Jean Baptiste Hardy de Lévaré3. Implantée dans le Maine depuis le xvesiècle, cette famille appartient au monde de la magistrature : «Ce qu'il y a de constant est qu'elle a dès ses premiers commencements vécu noblement en occupant plusieurs états distingués dans la robe ». Au XviIIesiècle, les garçons de la famille se partagent entre le droit et l'Église parmi les enfants de René I (1662-1722), juge civil et de police du comté de Laval, deux prêtres ${ }^{4}$ et deux avocats, dont le premier maire électif de Laval, Ambroise-Jean (1700-1780); parmi les enfants de ce dernier, quatre se destinent à la prêtrise, deux sont avocats, dont René II. Les filles concluent des alliances avec les familles les plus riches de la ville (maîtres de forge, négociants), ou les plus élevées en dignité (aristocrates).

René II dispose donc d'une position en vue dans la ville et le comté, il est le descendant de cinq générations d'avocats et de magistrats, le fils du maire de la ville, le petit-fils d'un premier juge; sa famille est également honorable par le nombre important d'ecclésiastiques qu'elle comporte. Lui même est né en 1738, il fait des études de droit, est avocat au Parlement de Paris. Quelques allusions dans la correspondance montrent qu'il a vécu dans la capitale; sans doute a-t-il bénéficié pendant ses études de l'hospitalité de son oncle, curé de Saint-Médard. Il épouse en 1766 Marie-Anne Guédé du Bourgneuf, s'installe à Laval comme avocat, est naturellement admis dans la société du jardin Berset dans laquelle se retrouvent les notables de Laval ${ }^{5}$. En 1772, il est commis de la recette des tailles alors que le propriétaire de l'office est son cousin Julien-André Coustard de Souvré, mineur émancipé dont il est le curateur. Dès l'année suivante, il acquiert l'office et s'y fait recevoir $\left({ }^{6}\right)$, mais il doit affronter l'opposition des héritiers du jeune homme qui composent cependant en $1782^{7}$. Les difficultés qu'il a rencontrées pour entrer en possession de cet office expliquent la différence entre l'évaluation à 60000 livres faite en $1771^{8}$ et l'affirmation de Hardy suivant laquelle il lui en a coûté 150000 livres $\left({ }^{9}\right)$... Malgré l'importance de sa finance, cette charge n'est pas anoblissante, Hardy se définit d'ailleurs comme " de caste roturière "

4 Les rôles fiscaux établis en 1792 placent René Hardy parmi les 24 plus gros contribuables à la cote mobilière ${ }^{10}$. D'après l'inventaire réalisé à son domicile, dans des circonstances dramatiques $\left({ }^{11}\right)$, en août 1794 , le montant des meubles de sa communauté est de 4084 livres il ne s'agit ici que des " meubles meublant", aucun des autres postes composant habituellement l'actif des inventaires, notamment les dettes actives et les fonds de prisée de bestiaux (dont on sait par ailleurs qu'ils sont de 6000 livres), ne sont indiqués. Ses biens fonciers se composent de maisons de ville et d'exploitations agricoles $\left({ }^{12}\right)$; on peut en dresser le tableau suivant, dans lequel ont été distingués les acquêts de la communauté (cté) et les biens propres, de la ligne maternelle (PM) ou paternelle (PP).

\begin{tabular}{|c|c|c|c|}
\hline $\begin{array}{l}\text { Localisation } \mathrm{du} \\
\text { bien }\end{array}$ & Désignation du bien & Nature & $\begin{array}{l}\text { Évaluation du bien } \\
\text { en mai } 1798\end{array}$ \\
\hline
\end{tabular}




\begin{tabular}{|c|c|c|c|}
\hline Laval & Maison et cour & PP & $7000 \mathrm{~F}$ \\
\hline Laval & $\begin{array}{l}\text { Maison occupée par Hardy, rue de la } \\
\text { Trinité }\end{array}$ & PP & $16000 \mathrm{~F}$ \\
\hline Laval & Maison rue du Val de Maine & PP & $800 \mathrm{~F}$ \\
\hline Avesnières & Deux maisons rue de Rivière & Cté & $1600 \mathrm{~F}$ \\
\hline Avesnières & $\begin{array}{l}\text { la closerie de la Grande Selle ou Mondésir } \\
\text { et les prés situés en Avesnières }\end{array}$ & $\begin{array}{l}1 / 3 \quad \text { cté } \\
2 / 3 \mathrm{PM}\end{array}$ & $12300 \mathrm{~F}$ \\
\hline Saint-Berthevin & la closerie de la Petite Bézière & PP & $8000 \mathrm{~F}$ \\
\hline Saint-Berthevin & la closerie de la Grande Bézière & PP & $7600 \mathrm{~F}$ \\
\hline Saint-Berthevin & deux closeries aux Brûlés & $\begin{array}{l}1 / 2 \quad \text { cté } \\
1 / 2 \mathrm{PP}\end{array}$ & $6000 \mathrm{~F}$ \\
\hline Bonchamps & la métairie du Bois Morin & Cté & $30000 \mathrm{~F}$ \\
\hline Astillé & la métairie de la Herberdière & PM & $24000 \mathrm{~F}$ \\
\hline Astillé & la métairie de la Pilaronnière & PM & $9000 \mathrm{~F}$ \\
\hline Le Genest & la closerie de la Louchère & PP & $5000 \mathrm{~F}$ \\
\hline Le Genest & 4 closeries de l'Écottière & PP & $15500 \mathrm{~F}$ \\
\hline Forcé & la métairie du Vau & PP & $8000 \mathrm{~F}$ \\
\hline
\end{tabular}

5 Cette fortune est caractéristique des notables bourgeois de Laval. Ils possèdent en ville une maison confortable qu'ils occupent et d'autres qu'ils louent; parmi leurs exploitations agricoles, de nombreuses closeries (exploitations de petite taille), dont certaines sont sans doute jointives (les 4 closeries de l'Écottière) et quelques belles métairies. On remarque enfin que ces exploitations sont situées dans des paroisses proches de Laval, ce qui permet une exploitation en métayage, comme le suggèrent les 6000 livres de prisée de bestiaux qui entrent dans l'actif de la communauté. Dans un de ses mémoires, Hardy revient sur la composition de sa fortune, qui a changé lors de l'acquisition de l'office de receveur des tailles : pour le payer, il a vendu six métairies. L'office permettait donc d'espérer un revenu qui valait qu'on lui sacrifiât autant.

La traversée de la Révolution

6 On ne dispose d'aucun renseignement sur les sentiments de Hardy au début de la Révolution; toutefois, il se retire complètement des affaires publiques dès 1790 (année de ses dernières recettes), et ne prend aucune responsabilité dans la nouvelle administration, ni municipale, ni départementale, contrairement à de nombreux notables lavallois $\left({ }^{13}\right)$, son homologue Frin de Cormeré par exemple. Le personnage de Jérôme-Charles Frin de Cormeré mérite qu'on s'y arrête un instant : né à Laval en 1751, 
il est trésorier des guerres en 1783, puis receveur particulier des finances dans l'élection de Laval, charge absolument équivalente à celle de René Hardy, l'un étant chargé des années paires, l'autre des années impaires. Il est comme lui membre de la société du jardin Berset (adhésion 1773), mais fait partie en 1786 des membres fondateurs de la loge maçonnique aristocratique des Amis-Unis de Laval ${ }^{14}$. Membre du comité de surveillance de l'une des sections de la ville, il adhère au fédéralisme et est arrêté en octobre 1793... mais reprend ses fonctions de receveur des impositions directes en février 1796, devient receveur général du département en février 1797, est nommé maire de Laval en 1810 (il meurt en charge en 1813) ${ }^{15}$.

La fin d'un monde la liquidation des offices

7 La décision de l'abolition et du remboursement des offices est prise par la Constituante; dans un mémoire présenté au tribunal civil du département de la Mayenne, Hardy évoque cet épisode «L'acquisition de cet office, l'augmentation de finance, et les procès indispensables pour la conservation de ce même office, lui ont coûté plus de 150000 livres en numéraire [...] ainsi le citoyen Lévaré en perdant son office a perdu dans le même instant le fruit d'un travail assidu de 30 années, la dot de sa femme, partie de son patrimoine, et demeure chargé de beaucoup de dettes ${ }^{16}$. La suppression de la vénalité des offices paraissait conforme au nouvel ordre des choses, mais l'indemnisation des nombreux officiers et leur inscription, sous certaines conditions, sur « le grand livre de la dette publique » se fait lentement : les services de la trésorerie n'examinent les comptabilités des receveurs particuliers qu'en juin 1795. Hardy doit chercher des hommes de confiance pour s'occuper de cette affaire. Un certain nombre de courriers lui parviennent d'un nommé Poirier, employé à la trésorerie nationale qui intervient de 1795 au début de l'année 1797. Le correspondant le plus régulier et le plus fidèle de la famille de Lévaré est un certain Bidault, homme de loi d'origine lavalloise implanté dans la capitale. Il est son conseiller et son ami, comme en témoigne la lettre de Hardy du 26 mars 1796 : «Nous avons confiance en vous et nous avons besoin de secours." Mais le 9 septembre 1803, Bidault, âgé de 75 ans, prévient Hardy qu'il ne peut plus s'occuper de ses affaires il définit en même temps la tâche qui était la sienne: "Mon âge avancé et l'affaiblissement de mes facultés intellectuelles ne me permettent pas de continuer à me charger ici de l'instruction, suite et sollicitation de vos affaires contentieuses. »

8 Les anciens officiers doivent fournir à la trésorerie la preuve que tous leurs comptes sont arrêtés et soldés (lettre de Poirier, 18 juillet 1795$)^{17}$. Hardy n'obtient ce certificat du département que le 30 avril 1796, car il lui a fallu rassembler préalablement 13 pièces différentes, dont 10 grosses d'arrêts de l'ancienne chambre des comptes donnant quitus pour les exercices 1762 à 1788 (années paires), le compte de l'impôt des chemins, exercice de 1790, le compte de la contribution patriotique, exercice de 1790, qui sont ses dernières recettes. Cette formalité accomplie, il est sur le point d'être inscrit sur le grand livre de la dette publique, mais son fondé de pouvoir, qui hante pour lui les bureaux de la trésorerie, lui apprend qu'un obstacle est apparu, empêchant l'achèvement de la procédure, "une opposition ", évoquée pour la première fois le 22 avril 1796 (lettre de Poirier). Datant de l'époque du procès qui a opposé Hardy, acquéreur de l'office de receveur, aux héritiers du précédent titulaire, cette opposition a bien été levée en son temps, mais des traces subsistent sur lesquelles on lui demande de s'expliquer. Plus de six mois plus tard, et bien que Bidault annonce, le 11 février 1797, « vous avez une inscription sur le grand livre de la dette publique consolidée [... ] 
de la somme annuelle de 3992 livres » correspondant au capital de 79840 livres, l'opposition n'a pas été levée.

9 Apparaissent alors dans la correspondance des personnages qui semblent toutpuissants puisque c'est en définitive de leur bon vouloir que dépend le franchissement des obstacles successifs nécessaire pour obtenir satisfaction. Après avoir essuyé les refus du citoyen Nau « conservateur pour la vérification et réception des oppositions formées sur les paiements à faire au trésor public et liquidation des offices supprimés » et du citoyen Alain "agent du trésor public pour la suite des instances relatives aux dettes et créances de la nation et pour tous les actes judiciaires de la trésorerie nouvelle ", les procureurs de Hardy se pourvoient en son nom au tribunal civil du département de la Seine, lequel, dans son audience du 26 ventôse anV lui donne raison. Malgré cette décision de justice, "on ne veut toujours point à l'administration se contenter de la quittance que vous m'avez envoyée [...] et on prétendait exiger de vous [...] des justifications à l'infini " (Bidault, 4 mars 1797). Les talents de négociateur de Bidault s'exercent alors, non seulement auprès de la hiérarchie (il rencontre le conservateur des hypothèques), mais surtout auprès du personnel des bureaux, comme l'agent Alain qui semble devenu très favorable: «Il a goûté les raisons que je lui ai données pour vous, il m'a montré les meilleures dispositions». Ils se mettent d'accord sur une stratégie, « tout cela va se faire d'accord, j'en ai la parole de $\mathrm{M}$. Alain et j'espère en conséquence que sous moins d'un mois ce sera une affaire consommée » (Bidault, 4 mars 1797). Le 26 mai 1797 enfin, Bidault peut écrire à son correspondant: «La mention de l'opposition est maintenant rayée. »

L'émigration du fils et la succession des malheurs

10 La situation d'ancien officier n'avait rien d'exceptionnel, et Hardy n'a sans doute pas souffert plus que les autres des lenteurs de l'administration et de la dévaluation des moyens de paiement ; celle de père d'émigré est infiniment plus difficile à vivre. Michel Denis évalue l'émigration en Mayenne à un niveau faible (150 à 200 personnes) ${ }^{18}$. Le départ de Xavier Hardy date du 18 janvier 1792. Quoique nous n'ayons aucun témoignage direct de sa part et que toutes les informations le concernant soient présentées par son père, un certain nombre d'éléments objectifs se dessinent. Son état civil tout d'abord, il est né le 17 mai 1770, il était donc majeur lors de son départ, il est célibataire et "sans état ", puisque après ses études de droit il était sur le point de se faire recevoir au Parlement de Paris, lorsque celui-ci fût supprimé. Selon ses propos, « il s'ennuie depuis 18 mois qu'il y est sans occupation », au point que «le séjour de Laval lui était maintenant insupportable $\aleph^{19}$. Son esprit d'indépendance et ses relations difficiles avec son père semblent attestés dans une famille où l'autorité paternelle ne se discute pas. Dans les circonstances du moment, sa place était ailleurs et « les chemins de l'honneur » dont il assure son père « qu'il n'y avait point à craindre un seul instant qu'il s'en écartât jamais " passaient sans doute, pour lui, par l'émigration ${ }^{20}$.

11 Xavier Hardy est de retour à Paris en juillet 1800, avant l'amnistie consulaire. Les termes de la correspondance de Bidault (lettre du 28 juillet 1800) donnent l'impression d'un personnage incontrôlable et imprudent «il y a deux jours que nous avons eu une visite à laquelle nous ne nous attendions très certainement pas, c'est celle de votre $\mathrm{X}$ [avier] tombé chez nous comme une bombe à 6 heures du matin ». Son arrivée n'est sans doute pas du goût du vieil homme qui le reçoit, par amitié pour sa famille, mais qui doit être bien effrayé d'une telle audace : «Je ne vous dissimulerai pas que, vu le peu de discrétion qu'a mis votre Xavier dans la visite qu'il nous a faite très à l'improviste, il 
nous a mis et nous resterons dans une inquiétude plus forte que le plaisir de le voir, jusqu'à ce qu'il ait obtenu et montré une radiation définitive » (lettre du 7 août 1800). À la fin de l'année, il est de retour à Laval et Bidault peut féliciter ses parents « du retour de la brebis égarée » (lettre du 8 décembre 1800), après huit ans d'abscence, pendant lesquels on ne sait rien de lui.

Dès 1792 , lorsque l'émigration du fils est connue $\left({ }^{21}\right)$, toute la famille est inquiétée ${ }^{22}$. Elle subit tout d'abord les dispositions relatives à l'assignation à résidence, en particulier l'arrêté du département de la Mayenne du 23 août 1792, conséquence de la loi du 15 août 1792, qui «consigne les père et mère femmes et enfants des émigrés dans leur municipalité sans aucune exception ». Hardy père est convoqué le 2 septembre 1792 par le procureur de la commune, " pour entendre la lecture de la loi du 15 août ». ${ }^{23}$

13 Par la suite, en septembre 1792, on lui demande de fournir l'équipement de deux gardes nationaux. Le 22 septembre 1792, le notaire Urbain Hubert, procureur syndic du district, lui signifie cette obligation, car « le départ de notre cavalerie nous force d'user de cette mesure sans aucun retard. Je vous prie donc de fournir deux manteaux de drap bleu dans le courant de la semaine prochaine à raison de chacun de vos fils émigrés [...] nous les ferions faire à nos frais si vous ne nous les donniez pas ». Le 22novembre 1792, son ancien homologue co-receveur Frin, désormais trésorier du district de Laval, prévient Hardy que « la solde et équipement sont fixés pour chaque enfant émigré à 881 livres 15 sols 8 deniers et il m'est ordonné d'en poursuivre le recouvrement ». Dès ces premières attaques, et cette attitude sera fréquemment la sienne lors de tous ses conflits avec l'administration, René Hardy ne s'adresse pas aux autorités locales, mais tente d'atteindre directement des sphères plus élevées. Il écrit au ministre de l'Intérieur Roland qui lui répond le 21 décembre 1792, sans doute pas dans le sens souhaité : «Puisqu'il vous est impossible de justifier de la résidence de l'un de vos fils dans la République, il faut que vous payez les 881 livres 15 sols 8 deniers qui vous sont demandées... »

Les lois condamnent les parents d'émigrés à l'internement (25 août 1792), et attribuent à la Nation la jouissance de leurs biens (11 janvier 1793). Les étapes de «la persécution " de la famille Hardy, telles que le père les raconte dans une pétition adressée le 11 septembre 1794 aux administrateurs du district de Laval, sont les suivantes : « On a mis en détention les père et mère le 26 mars 1793. On les a relâchés le 12 juillet suivant. On a repris le père seul le 12 octobre 1793, on l'a conduit à Chartres, on l'a ramené à Laval le 17 mai 1794. Le 26 juillet dernier, on a incarcéré son épouse et deux de ses filles et mis des gardes auprès d'une troisième fille malade le 13 août, on a rendu la liberté à sa fille ânée restent en détention le père, la mère et une fille. Du lieu de leur détention, les pétitionnaires viennent d'apprendre qu'on avait mis les scellées sur leurs effets mobiliers et fait saisir tous les revenus de leurs héritages de campagne, seule ressource pour la subsistance de toute la famille... » De fait, le 12 août 1794, le juge de paix réalise l'inventaire sommaire des biens mobiliers de René Hardy de Lévaré "à l'occasion de l'absence de son fils" et appose les scellés chez lui. La trace d'un paiement pour reconnaissance de scellés effectué par Hardy en novembre 1794, doit correspondre au moment où la famille a été libérée après un avis favorable de la commission philanthropique créée à Laval après thermidor ${ }^{24}$.

Depuis sa prison des Bénédictines de Laval, Hardy ne reste pas inactif et organise sa défense. Il s'appuie sur le sentiment que la loi du 7 décembre 1793 (17 frimaire an II), qui pose le principe de la saisie des biens des parents d'émigrés, fait une exception en 
faveur des parents d'émigrés majeurs qui prouveraient qu'ils ont agi activement pour empêcher l'émigration. Hardy va tenter de montrer qu'il se trouve dans ce cas il ne peut être considéré comme responsable des actes de son fils majeur, d'une manière générale, tous les deux étaient souvent en désaccord, et dans le cas précis de l'émigration, le père avait clairement dit à son fils qu'il désapprouvait cette attitude. Le 9 novembre 1794, il donne sa version des faits et fait comparaitre des témoins qui déposent tous dans le même sens ${ }^{25}$. Ainsi, il expose « que son fils aîné a quitté la maison paternelle en janvier 1792 à l'insu de ses père et mère que plusieurs années avant cette sortie ce fils indocile avait pris à tâche de désobéir en tout à son père que son père, apprenant qu'il voulait quitter la maison paternelle, lui défendit expressément de la quitter à peine d'encourir son indignation que ce fils a surpris un passeport de la municipalité à l'insu de ses parents sous prétexte d'aller à Paris qu'il n'a jamais approuvé ni devant ni depuis l'absence de son fils ceux qui émigraient, trouvant indigne qu'un honnête homme prît les armes contre sa patrie en conséquence il n'a donné aucun argent à son fils à cette époque. » Par ailleurs, Hardy rassemble différents indices de son "civisme », depuis lemariage de sa fille avec "un soldat républicain » jusqu'à des déclarations faites en privé témoignant de ses sentiments.

16 Parmi les témoins figurent, outre deux de ses amis (anciens officiers), des artisans ayant travaillé chez lui (une dresseuse et un maçon), des voisins et deux amis de la famille (un officier de santé et un notaire devenu officier municipal), ainsi que deux amis de son fils, dont un lieutenant de la garde nationale d'Ernée. Les mauvaises relations entre le père et le fils sont connues de ceux qui fréquentent la famille; d'une manière générale, tous témoignent du mauvais caractère du fils, de son esprit indépendant et du refus d'accepter les conseils de son père. Il peut paraître audacieux pour René Hardy de se faire passer pour favorable à la Révolution, il le tente cependant et trouve des témoins pour dire qu'il en parlait "d'une manière avantageuse ", du moins «dans le commencement». Plusieurs personnes témoignent l'avoir entendu appeler à respecter les lois en toutes circonstances, en particulier lors du tirage du 10 mars, où "beaucoup de gens vinrent le consulter », notation intéressante qui donne une idée de l'influence de ce notable et de la confiance de la population à son égard. Tous témoignent en tout cas de son «horreur » de l'émigration, assurent que celle de son fils s'est non seulement faite sans son consentement, mais sans qu'il en ait eu connaissance ; c'est ce que Hardy « a toujours dit à tous ceux qui ont voulu l'entendre ». C'est ce qu'il fallait démontrer, pensait Hardy, pour bénéficier du régime de faveur qu'on ne manquerait pas de faire aux parents d'émigrés qui s'étaient opposés à l'émigration de leur fils, suivant la loi du 7décembre 1793 (17 frimaire an II).

17 La remise en liberté ne met pas fin aux tracas en ce qui concerne les biens fonciers, au contraire, les ennuis commencent. Le 6 décembre 1794, on procède à leur adjudication, après séquestre, devant Louis Jean Moreau la Noë et Jean Joseph Barbeu, administrateurs du district de Laval. Ce dernier était, sous l'Ancien Régime, lieutenant général du siège ordinaire et membre de la Société du jardin Berset, comme tous les magistrats de la ville et comme Hardy lui-même. On peut imaginer qu'il ait permis ou encouragé des procédures permettant à Hardy, dans un contexte politique attentiste où la levée définitive du séquestre pouvait intervenir d'un jour à l'autre, de conserver la gestion de ses biens. Ses deux filles se rendent en effet adjudicataires de la majorité des exploitations qu'elles prennent à ferme, les exploitants restant en place, de telle sorte que le père peut, sous leur nom, jouir de ses revenus. Dans un premier temps, il a sans doute cru pouvoir le faire sans acquitter les fermages dus à la Nation; c'est 
vraisemblablement pourquoi il a payé, comme lorsqu'il était propriétaire, les impôts fonciers de 1794 et 1795, pour 46558 livres, ainsi que 3000 livres d'emprunt forcé. Mais au printemps 1796, la Nation lui demande une nouvelle contribution à l'emprunt forcé pour la somme de 3700 livres, payable en valeur métallique, et des fermages, payables en nature... L'effondrement de la monnaie ajoute au sentiment d'injustice : «Si je suis obligé de réaliser le prix des quintaux je vais être obligé d'acheter plus de 500000 en papier et ensuite quand me paiera-t-on de l'année 1794 et de l'année 1795, ce sera sans doute en papier qui plus on va moins ont de valeur » (Hardy, 28 mars 1796).

C'est alors qu'Hardy reprend contact avec d'anciennes relations parisiennes, membres de l'» ancien barreau», qui ont renoué avec les affaires et retrouvé des occasions d'exercer leurs compétences. Si le premier de ses conseillers reste Bidault, auquel Hardy expose régulièrement ses soucis, il échange également des courriers avec des personnalités comme Maucler, ancien avocat au Parlement de Paris, Lechat apparenté à la famille du conseiller au Parlement de Bretagne qui avait une résidence en Mayenne ${ }^{(26)}$, Duchauffour. Dans une lettre du 5 juin 1799, celui-ci évoque ses relations anciennes avec Hardy de Lévaré : « Depuis longtemps mon bon ami nous avons été dans le cas de ne pas communiquer ensemble [...] j'ai appris avec douleur que vous étiez encore exposé à des discussions dont l'effet doit affecter votre fortune... " Il donne des informations sur sa propre situation «je suis obligé de travailler comme si je recommençais ma carrière [...] j'aurais bien désiré de pouvoir vous servir mais mes anciennes relations n'existent plus... » On en apprend davantage dans la suite de la correspondance, en particulier dans une lettre du 24 août 1803 : «J'ai trouvé le moyen de quitter la préfecture dont je ne pouvais plus suffire les travaux en obtenant la place de greffier en chef du tribunal de police [...] Je ne pouvais trouver mieux d'après ma santé qui ne me permet plus de travailler comme je l'ai fait depuis 50 ans.» Bourdereau, le gendre de Bidault, apparaît à la fin de la correspondance, dans une lettre du 24 avril 1805 : «J'étais ci-devant avocat au Parlement de Paris et depuis notre dissolution je n'en ai plus exercé les fonctions que pour rendre les services que je me fais un vrai plaisir de vous offrir. »

$19 \mathrm{Au}$ printemps 1795, Hardy écrit pétition sur pétition pour obtenir la levée des séquestres. Les textes qu'il envoie à ses conseils pour avis reçoivent un accueil mitigé : ils n'approuvent pas la franchise avec laquelle il s'exprime sur le rôle de ses filles. Dans un courrier du 28 avril 1796, Bidault semble même irrité : « Nous vous le répétons une bonne fois pour toutes, il n'y a pas dans le moment actuel de mémoire à présenter en votre nom, nous le pensons ainsi, c'est au nom de vos enfants adjudicataires des biens de l'administration qu'il faut le faire ; on sait bien qu'ils ne sont ici que vos prête-noms, mais les baux ne disent et ne peuvent dire cela. » Hardy fait de nouvelles pétitions s'inspirant des avis de Bidault (lettres du 15 juin 1795, du 13 avril 1796 et du 28 juin 1796) qui se réfèrent encore à la loi du 17 frimaire anII : il s'agit de montrer que le séquestre des biens est nul car la loi s'applique aux émigrés; il faut démontrer que le fils n'est qu'absent (que d'ailleurs cette absence est contraire à la volonté de Hardy, mais a nécessité la complicité des autorités municipales qui lui ont fourni un passeport).

20 Si ce discours est au point, reste à savoir à qui adresser la pétition. Dans un premier temps, Hardy s'adresse aux politiques. Ses correspondants parisiens sont partisans d'épuiser les démarches au niveau local avant de s'adresser aux autorités centrales: «Le mémoire doit être présenté aux autorités constituées sur les lieux. Vous me dites 
sur cela en avoir parlé aux administrateurs de votre département qui vous ont dit avoir défense de s'en occuper. Eh bien, si cela est, ils répondront d'un "passé à l'ordre du jour» motivé de leur défense et nous nous autoriserons ici de cette réponse pour présenter la pétition au ministre des Finances, de là au directoire exécutif et enfin au corps législatif... C'est quand nous en serons venus à ce point que nous pourrons parler utilement à MM. Plaichard et Lanjuinais » (Bidault, le 28 avril 1796). Hardy aurait plutôt la tendance inverse ; on se souvient de sa lettre au ministre de l'Intérieur Roland, on trouve dans sa correspondance un billet de Lanjuinais, daté du 17 avril 1796, alors qu'il est membre du Conseil des Anciens. Il répond à une requête : « Votre pétition ci-jointe est de la compétence de l'administration du département. Il faut d'abord se pourvoir devant elle. Le recours ensuite est ouvert en cette matière au ministre des Finances qui ne manquerait pas de vous rendre justice. Je ne puis que vous donner cette instruction, compatir à vos peines et vous exprimer mes regrets de n'être pas à lieu de vous être utile. » Hardy le connaît-il, a-t-il eu l'occasion de le rencontrer à Rennes, lui écrit-il simplement en raison de ses opinions? Aurait-il écrit à Lanjuinais sans en prévenir ses amis parisiens? Bidault peut triompher dans un courrier du 28 juin : « Je ne suis point du tout étonné que $\mathrm{M}$ de Lanjuinais vous ait renvoyé votre mémoire » ...

Dans un second temps, il s'adresse aux juges, malgré ses réticences : "Suivant ce que vous mandez, le tribunal civil de votre département à commencer par le président n'est point composé de gens de loi et qui aient l'habitude des affaires ; c'est, je crois, un petit malheur, parce que nous devons toujours leur supposer de l'honnêteté et une bonne judiciaire; au surplus vous iriez par appel à un autre tribunal voisin de département et ensuite l'affaire viendrait ici au tribunal de cassation après quoi il y aurait encore à recourir au corps législatif » (Bidault, 27 juillet 1796). Bidault semble se résoudre à s'engager dans une procédure au long cours : «Il faudra bien essuyer, comme on dit, toutes les longues et les brèves et parcourir tous les degrés de juridiction (il y en aura 3 jusques et compris le tribunal de cassation où j'aurais quelqu'un à ma disposition pour le soutien et la défense de votre affaire... puis ensuite nous irions s'il le fallait par une pétition au Conseil des Cinq-Cents et de là à celui des Anciens... » (27juillet 1796). Suivant son conseil, Hardy adresse donc un mémoire au tribunal civil du département de la Mayenne (1er fructidor an IV, 18 août 1796) par lequel il demande la levée du séquestre sur ses biens, reprenant des thèmes évoqués dans les pétitions précédentes (différence entre l'absence et l'émigration, différence entre émigrés mineurs et majeurs, parents qui se sont opposés à l'émigration...). Le 29 août 1796 (12 fructidor an VII), le tribunal, expliquant que "les lois relatives aux émigrés... ont attribué aux administrations la compétence de tout ce qui est relatif au fait de l'émigration », se déclare incompétent et renvoie Hardy vers l'administration centrale du département ou vers le pouvoir exécutif... Malgré cet échec, Bidault ne semble pas ébranlé dans sa stratégie; il affirme à nouveau qu'il faut saisir le pouvoir judiciaire et suggère de recommencer l'action. Le 6 octobre 1796 (15 vendémiaire anV), il propose à Hardy un «nouveau travail», véritable cours de droit, à la fois leçon de procédure et catalogue des textes relatifs à l'émigration.

Et pourtant, le décret du 9 floréal an III (28 avril 1795), qui précise les conditions du séquestre des biens des parents d'émigrés, remet en cause cette démarche. Dès le 7 juin 1795, Maucler indique clairement à Hardy que ce texte ne permet plus d'invoquer l'exception qui paraissait appartenir aux pères et mères qui avaient résisté à l'émigration. Il faut désormais, selon lui, se résoudre à la déclaration des biens. Le long commentaire qu'il écrit pour Hardy sur la législation directoriale (joint à un courrier de 
Bidault du 11février 1797) se conclut ainsi : "le plan du gouvernement sur cette matière... se réduit à cette alternative, ou vous offrirez le partage de vos biens ou vous en souffrirez le séquestre ». L'hypothèse n'apparaît que le 21janvier 1797 dans une lettre de Bidault où il explique ses deux mois de silence (sa dernière lettre date du 18 novembre). "Il est des vérités que l'on n'aime pas, que l'on a toutes les peines du monde à se persuader à soi-même avant que de les persuader à ceux à qui il devient nécessaire de les dire, en se mettant pour le moment à leur place dans leur fâcheuse position, parce que ces vérités nous contrarient nous-mêmes dans les idées que nous avons de la justice et de la raison, et voilà pourquoi mon cher parent et ami j'ai été si longtemps à vous répondre... » Il donne le résultat de ses réflexions : « Au 20 floréal an IV il n'y a plus lieu d'espérer d'être favorablement écouté dans une demande de levée des séquestres, cette loi prévoyant pour les ascendants d'émigrés le maintien des séquestres ou le partage. »

Hardy se résout donc à demander le partage et présente pour cela une déclaration de tous ses biens meubles et immeubles et, en attendant que les opérations soient terminées, demande la levée du séquestre (pétition du 13mai 1797), ce à quoi l'administration centrale du département de la Mayenne consent. De fait, le 28 mai 1798, le partage avec la Nation des biens de Hardy de Lévaré, parent d'émigré, est réalisé : certains biens sont "réunis au domaine national ", sur les autres le séquestre est complètement levé. Hardy et son épouse «sont quittes envers le Trésor public, à raison de l'émigration de leur fils ». En août 1798, on procède à la vente aux enchères des biens confisqués, c'est-à-dire de la métairie du Vau de l'une des closeries de l'Écottière, de deux maisons rue de Rivière et de la métairie de la Pilaronnière, dont Hardy de Lévaré et son épouse se rendent adjudicataires.

Leurs soucis ne sont pas terminés pour autant, comme on peut le voir dans la pétition présentée à l'administration centrale du département de la Mayenne le 1er messidor de l'an VII (19 juin 1799) : Hardy est accusé de n'avoir pas déclaré tous ses revenus et est condamné à une forte amende. C'est sans doute en raison de cette nouvelle péripétie que sa closerie des Brûlés est de nouveau mise sous séquestre (Bidault, 9 septembre 1803). Ce dernier différend avec l'administration entraîne à nouveau Hardy dans le cycle des pétitions adressées à la nouvelle administration. Dès juin 1799, les mêmes questions se posent de savoir à qui faire parvenir les requêtes, à l'administration centrale (les conseillers d'État) ou locale (le préfet), qui faire intervenir... Les deux dernières années de la correspondance évoquent les nouvelles pétitions de Hardy, jusqu'à la mention dans une lettre de Bourdereau, le gendre de Bidault, le 24 avril 1805, du rejet d'une dernière demande par l'Empereur lui-même.

Une correspondanceDes correspondants d'infortune

Les correspondants de Hardy de Lévaré, s'ils ne partagent pas tous ses malheurs (en particulier l'émigration de son fils et le séquestre des biens familiaux qui en résulte), sont tous des avocats, pour la plupart d'entre eux d'anciens officiers, pour qui la Révolution a entraîné des modifications de style de vie considérables. Bidault le suggère à propos de la législation sur les offices: "Il est bon que vous sachiez et je vous l'apprends avec bien du plaisir, vu l'intérêt que nous y prenons tous que vous, et bien d'autres compagnons d'infortune êtes relevés de la déchéance que les précédentes lois vous avaient fait encourir. Il y avait longtemps que nous attendions ce remède à nos maux, tout depuis le 9 Thermidor dernier nous le faisait espérer " (Bidault, 15 juin 1795). 

l'objet de ces échanges épistolaires : «Vous avez mon cher parent et ami le chagrin dans le cœur et moi aussi » (Bidault, lettre du 22février 97) ; le sentiment dominant est souvent l'impuissance devant le sort qui semble s'acharner. Ces personnages ne sont pas dans les bons réseaux, « et malgré qu'il n'y ait rien de bien consolant à dire qu'on n'a ni accès ni connaissance auprès du ministre qu'il s'agirait d'intéresser à votre position, encore faut-il vous le dire ».Dans son courrier du 12 juin 1796 Maucler décline successivement lassitude: "Je ne vous répondrai pas mon ancien ami d'après ma propre doctrine sur des lois dont je n'ai jamais eu le courage de suivre la marche... ", scepticisme : «dans une matière rigoureuse on ne doit pas se flatter de faire admettre des exceptions; quand on a pour soi la vérité des faits, on a le droit sans doute de la réclamer, mais il ne faut pas calculer sur leur succès ", réprobation : "il aurait fallu dans les derniers jours avoir des amis en enfer, c'est-à-dire parmi les agioteurs et avoir du numéraire pour avoir des mandats à vil prix où ils étaient tombés. On nous dit qu'ils ont repris valeur quadruple aujourd'hui quoiqu'ils ne soient pas encore au sixième de leur valeur nominale. Mais il $\mathrm{y}$ a un genre de succès auquel l'honnêteté ne peut aspirer. »

La majorité de ces personnages ne sont pas dénués d'humour. Maucler en particulier attend dès le 7 juin 1795 «que de bons esprits aient trouvé la sortie de la caverne où nous sommes enfoncés ». Le même personnage fait une analyse partiale mais lucide de la loi du 9 floréal an III, qui a ordonné le partage des biens des parents d'émigrés... " mais bientôt ce partage exigé d'autorité des pères et mères vivants apparut trop brutal et la loi a été suspendue. La suspension a cessé quand après une année révolue les législateurs ont découvert les moyens d'arriver au même but par une voie plus polie. La République a dit aux ascendants d'émigrés nous n'exigerons aucun partage par autorité mais nous voudrons bien l'accepter quand vous viendrez nous l'offrir. C'est ce trait de génie qui a enfanté la loi du 20 floréal anIV, loi qui loin d'être pénale est au contraire dit-on une loi de faveur puisqu'elle met dans la main des pères et des mères et autres ascendants d'émigrés l'usage d'une faculté au moyen de laquelle ils peuvent faire cesser le séquestre de leurs biens... Le plan du gouvernement sur cette matière se réduit à cette alternative : ou vous offrirez le partage de vos biens, ou vous en souffrirez le séquestre. Telle est la marche de l'autorité contre laquelle on lutterait inutilement. J'ai cru en conséquence qu'il fallait se présenter au partage, non par le motif de la persuasion, mais parce qu'il faut se laisser conduire par la leçon de l'expérience » (11 février 1797).

Sur dix années de correspondance (1795-1805), même si la situation des protagonistes et leurs difficultés ne sont que « le contrecoup de celles de la chose publique » (Bidault, 27 juillet 1796), on trouve peu d'allusions précises au contexte politique. Pour les régions de l'Ouest en général et le département de la Mayenne en particulier, les opérations armées sont le premier souci. Les revenus de l'année 1795 ont été médiocres: "Les héritages ont beaucoup souffert du passage des troupes et des incursions des gens armés. Les bras manquent à l'agriculture. La Nation a mis en réquisition fourrages, bestiaux et grains » (pétition d'avril 1796). Les «chouans » sont encore présents au printemps 1796. Les troubles de 1800 apparaissent plus graves encore; pour la première fois Bidault en évoque la conséquence sur les communications : « Les troubles qui agitent vos malheureuses contrées et qui sont à la veille de prendre le caractère le plus terrible ne vont-ils pas faire obstacle à notre

Annales historiques de la Révolution française, 323 | janvier-mars 2001 
correspondance ?» (Bidault, 15 janvier 1800). Un mois plus tard (14 février) il est plus optimiste: "Nous croyons ici d'après les journaux pouvoir vous féliciter tous du rétablissement de la paix dans votre ville et les environs... " Satisfecit rare dans cette correspondance, Lechat félicite le gouvernement: «J'ai souvent pensé à votre pénible situation au milieu des troubles qui agitent les départements de l'Ouest. Grâce au ciel, ils vont entièrement cesser, puisque notre gouvernement actuel réunit le pouvoir à la volonté... »(1 $1^{\mathrm{er}}$ janvier 1800).

On rencontre également quelques allusions à l'attente des élections sous le Directoire. Bidault, le 26 mai 1797, évoque « le renouvellement du corps législatif qui fait concevoir des espérances pour l'adoucissement des lois portées contre les familles des émigrés et absents ». Le 3 juillet 1799, après avoir formulé des vœux pour la réussite des affaires de Hardy, il espère «que la nouvelle tournure de la chose publique n'y aura point influencé en mal... ».

Loin des cénacles parisiens, mais témoignant de la considération dont Hardy pouvait jouir dans les milieux catholiques conservateurs, le curé d'Athée, fraîchement revenu d'exil, souhaite connaître son sentiment à propos de la promesse de fidélité à la Constitution de l'an VIII exigée des prêtres: «Marquez-moi je vous prie ce que vous pensez des affaires concernant la promesse et ce qui se fait chez vous à cette occasion. Je trouve qu'il est fort difficile de prendre un parti... M. Chatizel ${ }^{27}$ donnerait de grandes lumières sur cet article s'il était en pays je m'en fierais bien à sa décision. Je serais encore plus content si Rome parlait. " (Vaulgeard ${ }^{28}$ curé d'Athée, 19 juillet 1801). Cette demande de soumission, qui figure dans une déclaration des consuls de décembre 1799, est rendue obligatoire par un arrêté du préfet de la Mayenne le 4 juillet 1801. Au moment où il écrit, les hésitations du curé d'Athée sur l'attitude de Rome ne sont plus de mise, puisque le Concordat est déjà signé (15 juillet 1801).

31 S'il y a peu de lignes consacrées à l'évolution de la situation politique et aux positions des uns et des autres, pratiquement toutes les lettres décrivent ou analysent le fonctionnement de l'administration et s'interrogent sur les moyens à mettre en œuvre pour en obtenir satisfaction.

Du bon usage de l'Administration

La première difficulté consiste apparemment à connaître le texte exact des lois. Bidault écrit le 15 juin 1795 : «Je ne puis rien vous dire de bien positif encore sur ce que vous devez faire pour obéir à la loi du 9 floréal, n'en ayant pas les dispositions précises et détaillées. J'en ai causé ce matin avec le citoyen Poirier votre fondé de procuration que j'ai vu et qui n'est guère plus avancé que moi là-dessus. "

33 L'incertitude alimente les interprétations et les spéculations, comme le suggèrent les formules utilisées par Maucler dans une lettre du 7 juin 1795, à propos du décret du 9 floréal anIII : «on tient pour certain... », « on doute... ", « le décret semble supposer... ", "plusieurs espèrent cependant... ". Plus tard, le 12 juin 1796, le même Maucler évoque les discussions qu'on imagine très intéressées, à propos de «la nouvelle loi » (sans doute du 9 mai 1796) dans les milieux où les proches d'émigrés sont nombreux "on tient assez communément... ", " on croit également... ", "l'opinion la plus générale est que... », « on prétend ici... ». Des délais entre la loi et son application dans les départements font l'objet de commentaires optimistes : «ceux qui voient en beau ajoutent que ce retard a pour objet de diminuer la rigueur de la loi... » modifient, comme l'explique Maucler à propos du décret du 9floréal an III : « On est 
persuadé qu'il interviendra quelque modération aux rigueurs du décret, on en cite même une déjà comme à peu près convenue... Comme quand une fois la nécessité de quelques exceptions aura été reconnue, l'esprit de justice peut conduire de l'une à l'autre, la conclusion ordinaire est qu'il est bon de profiter du délai du décret et de ne donner sa déclaration que dans un temps voisin de son expiration en se tenant toujours en mesure pour y satisfaire. » Il faut donc savoir utiliser les délais, ne pas se précipiter de manière à pouvoir profiter d'une éventuelle disposition plus favorable. Dans une lettre du 13 avril 1796, Bidault semble modérer l'impatience de son ami : « Vous devez rester tranquille et ne rien dire... lorsqu'il y aura une loi claire et précise à cet égard (des parents des émigrés) vous vous montrerez. » Une telle attitude conduit parfois à placer les individus dans un état de tension permanent. Bidault écrit de Fontenay-auxRoses, le 28 juin 1796 : « les circonstances des temps sont telles que continuellement on en est aux expédients sur ses propres affaires, on est aussi perpétuellement sur le quivive ne sachant jamais le jour ce qui arrivera le lendemain. Nous avons hier soir quitté Paris pour venir ici où nous avons un petit logement à loyer dont nous ne faisons pas un grand usage étant sans cesse ramenés à Paris par la crainte que notre absence ne nous y mette en défaut. » Tous les correspondants de Hardy le disent, il est d'autant plus important de se tenir au courant des nouvelles dispositions qu'il faut être toujours prêt à s'y soumettre : «Il faut vous attacher à l'exécuter dans la plus grande ponctualité en vous conformant tout à la fois au texte et à l'esprit de la loi » (Bidault, 15 juin 1795).

Aussi importante que la bonne connaissance des textes eux-mêmes, celle de «ce que l'on appelle l'air du bureau " (lettre de Bidault, le 17 juin 1799). Le terme même de bureau est très fréquemment employé, pour qualifier un type d'organisation, avec ses employés, ses divisions et ses chefs, mais aussi un certain type de fonctionnement. Successeurs des bureaucrates d'Ancien Régime ${ }^{(29)}$, ceux de l'époque révolutionnaire constituent un pouvoir distinct du politique. "Nous disons, M. Maucler et moi, que c'est affaire de bureau, que c'est ainsi que l'on y a réussi à Paris en pareille circonstance, en négociant avec l'administration centrale et se donnant bien garde de recourir pour cela aux autorités supérieures. » Il semble que, paradoxalement, dans le fonctionnement des bureaux, les individus ont une grande importance. Bidault explique comment court-circuiter la hiérarchie ordinaire : "Nous avons été retrouver le citoyen Alain qui doit revoir le ministre et fera humainement tout le possible pour avoir de lui, sans l'entremise du chef de ses bureaux en cette partie, un mot d'écrit pour vous » (3juin 1799). La correspondance présente aussi des personnages moins aimables, tel un certain Pajot : « Hier je me suis rendu rue de Tournon Hôtel de Minervois pour y voir M. Pajot et lui montrer votre lettre... il m'a alors été dit que M. Pajot n'était pas encore à son bureau, qu'il ne tarderait pas à y arriver, mais que ce n'était pas jour à pouvoir lui parler par ce qu'il ne recevait le public que les mercredi et samedi... Réfléchissant à l'urgence... je prenais le pari d'attendre qu'il arrivât et il n'a pas tardé je me suis en conséquence hasardé à lui parler malgré que l'on m'eût répété plusieurs fois que ce n'était pas jour à le voir et effectivement j'ai été de lui reçu comme, dit le proverbe, un chien dans un jeu de quilles ... » (Bidault, 16 septembre 1803).

Pratiques caractéristiques des hommes de loi d'Ancien Régime habitués à la valeur des protections et qui cherchent à reconstituer leur univers ou évidente continuité des usages entre l'ancienne administration et la nouvelle, la recherche de bons intermédiaires reste essentielle au succès d'une affaire. On sollicite des personnages que l'on connaît. Poirier, le fondé de procuration de Hardy, va faire intervenir Lechat, « toujours rempli de bonne volonté pour vous et nous espérons que, par son moyen, il 
parviendra enfin à obtenir la liquidation de la finance de votre office» (lettre de Bidault, 15 juin 1795). Quatre ans plus tard, le même personnage a conservé toute son influence: "S'il en résulte qu'il faille obtenir quelque mot encore du ministre des Finances, mandez-le-moi, je m'adresserai aussitôt... à M.Lechat... » (Bidault, le 17 juin 1799). Il ne s'agit parfois guère plus que de conseiller des adresses. Alors qu'il a demandé le partage de ses biens, une question préoccupe Hardy, qui est de savoir si la Nation peut prendre dans son lot « l'inscription sur le grand livre ». Consulté, Maucler répond: «Je vous dirais de vous adresser au citoyen Boivin rue des Grands-Augustins $\mathrm{n}^{\circ} 30$ qui a fait réussir à ma connaissance des opérations de cette nature en l'avertissant de m'en parler il ne s'y refuserait pas... » (20 janvier 1797). Mais ne connaître personne dans une administration, voire un tribunal auquel on s'adresse semble dramatique: Bidault évoque le 20 octobre 1797 « La malheureuse circonstance où vous vous trouvez de ne connaitre personne [...] dans aucune des communes aux tribunaux desquelles vous seriez dans le cas de vous portez appelant »; Hardy écrit à un administrateur de l'enregistrement et des domaines, le 4 mars 1805: «Si vous avez la bonté de faire passer un petit mot de recommandation à ce ministre [...] je vous avoue que je ne [le] connais point, ni personne que je puisse employer auprès de lui. »

37 À travers cette riche correspondance, on voit se reconstituer un réseau d'avocats des anciens barreaux et particulièrement du barreau de Paris. Ces personnages sont d'âge divers; les plus âgés ou les plus riches semblent avoir cessé leur activité professionnelle, mais continuent à donner des consultations et à s'entremettre, d'autres entreprennent de nouvelles carrières, dans les nouveaux tribunaux ou les nouvelles administrations. Bien qu'on ait le sentiment qu'ils regrettent tous une grandeur passée, évoquant des logiques qui n'opèrent plus ou des relations qu'ils n'ont plus, certains d'entre eux semblent retrouver une certaine influence. L'accélération de l'activité législative et réglementaire et le développement de l'administration qui l'accompagne sont tels que les hommes de loi de l'Ancien Régime y réintroduisent leurs compétences et leurs pratiques.

Les mésaventures de Hardy de Lévaré ne sont pas isolées et sur les listes établies pour le département de la Mayenne, on compte les émigrés par dizaines. Les «fils de famille émigrés» de Laval en revanche ne sont que sept sur la liste arrêtée en juin $1792^{30}$. Parmi les familles dont les propriétés ont été séquestrées en raison de l'émigration d'un de ses membres on retrouve beaucoup de noms de familles de l'aristocratie et celui de quelques rares roturiers dont précisément celui de Hardy de Lévaré. C'est peut-être en cela que le destin de René Hardy est original : pendant la Révolution il a, à cause de l'émigration de son fils, un destin d'aristocrate, alors que ni lui, ni aucun des membres masculins de sa famille, ne se sont anoblis sous l'Ancien Régime. Cette situation s'explique peut-être par l'insuffisance de leurs moyens, mais plus certainement par l'attachement à la carrière du barreau et à la ville de Laval (dont aucune charge n'est anoblissante) ainsi qu'à un certain nombre de valeurs essentiellement bourgeoises, répétées à l'envi par celui qui fait figure de patriarche, Ambroise-Jean, le premier maire électif de Laval, le propre père de René Hardy. Parmi ces valeurs, l'économie, le travail, le sens du devoir, le respect de la tradition, la crainte et l'amour de Dieu. La correspondance familiale dans laquelle se détache également la figure de l'oncle de René Hardy, curé de Saint-Médard, violemment opposé aux philosophes comme aux jansénistes, et condamné par le parlement pour refus de sacrement ${ }^{31}$ ), donne le sentiment d'une critique permanente des idées du siècle. Deux extraits de lettre adressée à un autre jeune homme de la famille qui se destine à la profession d'avocat 
par Ambroise-Jean Hardy, son grand père, d'une part et par René Hardy son oncle permettent de comprendre l'attachement forcené de cette famille à la tradition. Le grand-père écrit à son petit-fils qui vient d'être reçu avocat au Parlement de Paris, le 14 février 1772, « quand on s'établit en province, il faut se conformer au pays où l'on est et ne pas conserver des idées de grandeur; vous apprendrez avec moi la simplicité et l'humilité, ce sont les leçons que j'ai reçues de mon père et que je n'ai cessé et ne cesserai de vous donner " "2 $^{32}$ De son côté, René Hardy écrit à son neveu : "Nous tenons de nos pères deux trésors l'un est l'amour de la vertu et l'autre l'amour du travail, les deux avantagesne peuvent subsister l'un sans l'autre, nous faisons tous nos efforts pour ne point dégénérer venez en partage avec nous, montrez que réellement vous êtes de notre famille et que vous en avez tous les sentiments. » (4janvier 1769) ${ }^{33}$

Une des explications au repli de la famille en 1789-1790 pourrait bien être son attachement à un catholicisme très rigoureux. Sans doute René Hardy était-il trop prévenu par son éducation pour être favorablement touché par le souffle révolutionnaire et son retrait des affaires alors que bien des notables lavallois, qu'il côtoyait au jardin Berset, s'engagent dans la nouvelle administration, témoigne de ses réserves précoces sur le nouveau cours des choses. Par la suite, son attention aux questions religieuses demeure; parmi sa correspondance figure un billet dont il a dû apprécier le ton peu amène pour le clergé constitutionnel et qu'il a recopié de sa main : "Cytoyen, je vous adresse une pièce qui vous fera connaitre les singes de l'église catholique ; c'est aujourd'hui que Lecoz prétend faire de Dorlodot un évêque " ${ }^{34}$.Alors que ses affaires s'améliorent, la correspondance évoque des services rendus au Lavallois par les Parisiens, notamment par un des fils de son ami Bidault, libraire, qui lui procure des revues et des livres. On voit même, en 1805, Hardy chercher à faire publier différents ouvrages, l'un relatant "le loisir des prêtres de Londres", l'autre qui pourrait prendre la forme de "dissertations théologiques » sur l'eucharistie, sur saint Joseph, sur Ponce-Pilate... dont les auteurs n'ont comme but que de "réveiller l'attention de ceux qui aiment la religion » et qu'Hardy souhaiterait présenter au pape. On comprend donc, même si la correspondance est muette sur le sujet, que la politique religieuse des autorités révolutionnaires n'ait pu trouver grâce à ses yeux.

Cet état d'esprit se maintient-il à la génération suivante? Il y a évidemment dans l'émigration du fils une part de conviction (il s'engage à nouveau dans la chouannerie en 1815) mais elle semble aussi être l'aboutissement de la conjonction de facteurs favorables (l'âge, l'inaction, la disponibilité, la mésentente avec le père, la recherche de l'aventure). Sa carrière semblait toute tracée: avocat, il aurait acquis une charge quelconque et aurait suivi l'exemple de son père, de son grand-père, de ses oncles. C'est par d'autres moyens et avec des conséquences dramatiques pour sa famille que Xavier Hardy va lui aussi faire tous ses efforts « pour ne point dégénérer » 


\section{NOTES}

1.Lettre du diacre Hardy à sa mère, s.d., Archives départementales de la Mayenne (noté désormais ADM), $17 \mathrm{~J} 102$.

2.Les fonds Hardy rassemblent des documents provenant de plusieurs membres de cette famille de notables, et particulièrement de trois personnages, appartenant à trois générations successives René I Hardy de Lévaré (1662-1722), juge de police et juge civil du comté pairie de Laval, ADM 14 J 45, son fils Ambroise-Jean (1700-1780), juge de police puis maire électif de Laval, ADM $17 \mathrm{~J}$, et son petit-fils René II (1738-1815), receveur particulier des finances de l'élection de Laval, ADM $14 \mathrm{~J} 48$.

3.ADM, 1Mi 146, R 29. Jean-Baptiste Joseph Hardy (1702-1765), avocat, est le fils de René $\mathrm{I}$, le frère d'Ambroise-Jean et l'oncle de René II.

4.René Florent (1690-1736), curé de l'Huisserie et Pierre (1696-1778), curé de SaintVénérand de Laval en 1739, puis chargé de la cure de Saint-Médard à Paris en 1742 voir Jules-Marie RICHARD, "Lettres lavalloises, Bulletin de la Commission historique et archéologique de la Mayenne (BCHAM), 1911 et 1912.

5.En 1766, la société enregistre l'adhésion de "Hardy... ADM $183 \mathrm{~J} 18$ voir Jules-Marie RICHARD, "La société du jardin Berset à Laval, BCHAM, 1910.

6.Provisions accordées par le Roi le 4 août 1773 réception par les officiers de l'élection le 30 août, ADM C 287.

7.Acte Leray 12 août 1782, ADM 3 E 35/214.

8.Évaluation de l'office de receveur de la taille, 23 octobre 1771, ADM 3 E 35203.

9.Mémoire au tribunal civil du département de la Mayenne, 18 août 1796, ADM $14 \mathrm{~J} 48$. 10.Frédérique PITOU, Laval au XVIIIe siècle, Marchands, artisans, ouvriers dans une ville textile, SAHM, Laval, 1995, p. 144.

11.Inventaire du citoyen Lévaré à l'occasion de l'absence de son fils, 12 août 1794, ADM $14 \mathrm{~J}$ 48. Ilest réalisé par un juge de paix en vertu d'ordres du district.

12.Extrait des registres des arrêtés de l'administration centrale du département de la Mayenne, 9prairial an VI, ADM $14 \mathrm{~J} 48$.

13.Listes dans Ferdinand GAUGAIN, Histoire de la Révolution dans la Mayenne, Mayenne, 1989, 4vol. (1re éd. 1918), t. I, pp. 175 et 523.

14.André BOUTON, et Marius LEPAGE, Histoire de la franc-maçonnerie dans la Mayenne

(1756-1951), Le Mans, 1951.

15.F. GAUGAIN, La Révolution..., t I, pp. 344 et 345.

16. Mémoire au tribunal civil du département de la Mayenne, 18 août 1796, ADM 14 J 48. 17.Toutes les lettres citées se trouvent en ADM $14 \mathrm{~J} 48$, sauf indication contraire.

18.Michel DENIS, Les royalistes de la Mayenne et le monde moderne, XIXe-XXe siècles,

Klincksieck, 1977, p. 83. Xavier Hardy de Lévaré figure dans le tableau des anciens émigrés de la Mayenne en 1815, p.87.

19.Lettre de Xavier Hardy à ses parents, du 18 janvier 1792, déposée par son père chez le notaire Aubry, 3 E 40150. C'est le seul document que nous possédions de la main de Xavier Hardy il est écrit à Laval avant son départ et ne dit évidemment rien de ses intentions.

20.“L'émigration était générale, à cette époque écrit le général d'Andigné en résidence à Angers chez sa mère puis au château de Princé chez sa sœur, en 1791, Mémoires du Général d'Andigné, Mayenne, Yves floch, 1990, t 1, p. 90 (1re édition Paris, Plon, 1900). 
21.Contrairement à ce qu'affirme son père, “jamais Xavier Hardy Lévaré n'a été compris dans aucune liste d'émigrés, c'est un fait de notoriété publique, il figure bien dans les différentes listes dressées depuis juin 1792, ADM Q 88, Q 100,

Q 101, Q 102, Q 103.

22.Hardy de Lévaré et son épouse figurent notamment dans la "Liste des particuliers indiqués pour la réclusion dans la maison des Bénédictines, 26 mars 1793 (ADM L 1319) qui leur vaudra leur arrestation et dans l'“État nominatif des parents d'émigrés et de leurs alliés, des ci-devant nobles compris dans les lois des 3 brumaire an IV et 9 brumaire an VI, des aïeuls et aïeules de chouans... dressé en exécution de la loi du 24 messidor an VII sur la répression de la chouannerie (ADM L 481).

23.Un registre est alors ouvert "pour y recevoir la signature des parents des émigrés qui se rendront chaque jour au secrétariat de notre municipalité, ADM L 612. Le registre est tenu du 5septembre 1792 au 2 avril 1793 et ne recueille qu'une dizaine de signatures, toujours les mêmes en tout cas celle de Hardy de Lévaré n'y figure jamais. 24. Registres de délibération de la commission philanthropique, brumaire-frimaire an III, ADM L 1629. Sur cette commission, Jean STEUNOU, 1790-1795, Laval, Siloë, 1990.

25.Enquête de témoins pour Hardy de Lévaré, du 19 brumaire an III (9 novembre 1794), ADM $14 \mathrm{~J} 48$.

26.Gauthier Aubert évoque la richesse de l'inventaire fait après le décès du conseiller Lechat en 1750 dans son château de Vernée, Gauthier AUBERT, "Nobles rennais en pays ligériens, La Mayenne, 1999.

27.Sans doute Pierre Jérôme Chatizel de la Néronnière (1733-1817), Alphonse ANGOT, Dictionnaire de la Mayenne, Mayenne, Floch, 1986, 4 t., t.1, pp. 612-613 et Émile-Pierre QUERUAU-LAMERIE, "L'abbé Chatizel de la Néronière, curé de Soulaines-en-Anjou BCHAM, t. XV, 1899.

28.Pierre-François Vaulgeard, curé depuis 1787, refusa le serment, fut exilé en Angleterre, reprit ses fonctions en 1800, mourut en 1813, A. ANGOT, Dictionnaire... article "Athée, t. 1, p. 92.

29.Daniel Roche évoque la montée de la "bureaucratie "La centralisation du xviIIe siècle produit les bureaux et les archives, les enquêtes et les formalités, et soulève des protestations rituelles contre l'engorgement et la paperasserie. En même temps, elle produit d'autres réalités, distribuant le pouvoir et arbitrant les conflits selon des règles qui ne sont plus tout à fait celles de la société inégalitaire..., Daniel RocHE, La France des Lumières, Fayard, 1993, p. 200.

30.ADM Q 100.

31.ADM $17 \mathrm{~J} / 167$ à 213 famille Hardy de Lévaré, correspondance familiale et professionnelle, 1688-1782.

32.ADM $17 \mathrm{~J} 190$.

33.ADM $17 \mathrm{~J} 191$.

34.Dorlodot est consacré comme évêque constitutionnel de la Mayenne le 7 avril 1799 par Le Coz, métropolitain de Rennes. 


\section{RÉSUMÉS}

La correspondance qui lui est adressée, essentiellement de 1795 à 1800, permet de retracer les mésaventures d'un notable contre-révolutionnaire. En 1789, René Hardy fait partie des plus grosses fortunes de la ville de Laval, il est avocat et pourvu de l'office de receveur des tailles. Il cesse toute activité publique dès 1790 , mais sa vie et celle de sa famille basculent avec l'émigration de son fils aîné, en 1792; elle entraîne son emprisonnement, la mise sous scellés de ses meubles, le séquestre de ses biens fonciers. Sans beaucoup de commentaires sur la situation politique, ses correspondants, essentiellement d'anciens avocats, consacrent toute leur énergie à comprendre la législation concernant les parents d'émigrés et à choisir la stratégie la plus efficace pour obtenir satisfaction de l'administration.

The Reluctant Aristocrat, or the Mishaps of an Émigrés Father (1792-1805).

The correspondence which is addressed to him, from 1795 to 1800 , makes possible to recount the mishaps of a leading-citizen during the Revolution. In 1789, Rene Hardy belongs to the largest fortunes of the town of Laval, he is a lawyer and owns one office of receiver of the taxes. He stops any public activity since 1790 , but its life and that of its family change with the emigration of his elder son, in 1792 ; it involves its imprisonment, the setting under seals of its furniture, the sequestration of its land properties. Without a lot of comments about the political situation, its correspondents, former lawyers, devote all their energy to understand the legislation concerning the parents of emigrants and to choose the most effective strategy to obtain satisfaction of the administration.

\section{INDEX}

Mots-clés : Contre-Révolution, émigration, avocats, Mayenne, Laval

\section{AUTEUR}

\section{FRÉDÉRIQUE PITOU}

Université du Maine (Laboratoire d'Histoire Anthropologique) 